Ciência Florestal, Santa Maria, v. 27, n. 4, p. 1269-1281, out.-dez., 2017

ISSN 1980-5098

\title{
MICORRIZAS ARBUSCULARES E FOSFATO NO DESENVOLVIMENTO DE MUDAS DE CEDRO-AUSTRALIANO
}

\section{ARBUSCULAR MYCORRHIZA AND PHOSPHATE ON GROWTH OF AUSTRALIAN RED CEDAR SEEDLINGS}

\author{
Emanuela Pille da Silva ${ }^{1}$ Paulo Ademar Avelar Ferreira ${ }^{2}$ Antônio Eduardo Furtini-Neto ${ }^{3}$ \\ Cláudio Roberto Fonsêca Sousa Soares ${ }^{4}$
}

\begin{abstract}
RESUMO
O cedro-australiano (Toona ciliata M. Roem var. australis) é uma espécie que tem apresentado potencial de utilização florestal no Brasil. Deste modo, o objetivo deste trabalho foi determinar a resposta de mudas de cedro-australiano à inoculação com fungos micorrízicos arbusculares (FMA) em diferentes doses de fósforo $(\mathrm{P})$. $\mathrm{O}$ experimento foi conduzido em delineamento inteiramente casualizado em esquema fatorial 8 × 2, com cinco repetições. Os tratamentos consistiram na inoculação de sete espécies de FMA: Rhizophagus clarus, Claroideoglomus etunicatum, Racocetra gregaria, Acaulospora colombiana, Acaulospora morrowiae, Gigaspora margarita e Dentiscutata heterogama, além de um tratamento sem inoculação; sendo submetidos a duas doses de fosfato, 25 e $250 \mathrm{mg} \mathrm{dm}^{-3}$ que equivaleram a teores de 10,7 e $41,3 \mathrm{mg}$ $\mathrm{dm}^{-3} \mathrm{P}$ no solo, respectivamente. Avaliou-se a porcentagem de colonização micorrízica, esporulação, altura, diâmetro, matéria seca da parte aérea e acúmulo de nutrientes, 150 dias após o transplantio. Houve diferença em função das doses de $\mathrm{P}$ e dos isolados nas variáveis avaliadas. Os isolados Claroideoglomus etunicatum e Acaulospora colombiana beneficiaram o crescimento e a absorção de nutrientes do cedro-australiano em baixa disponibilidade de $\mathrm{P}$, enquanto que Acaulospora morrowiae se mostrou mais promissor em solos com maior disponibilidade desse nutriente. Esses isolados proporcionaram elevada colonização micorrízica no cedro-australiano, favorecendo a produção de mudas de qualidade com vistas ao estabelecimento e produtividade de plantios comerciais.
\end{abstract}

Palavras-chave: associação micorrízica; adubação fosfatada; produção florestal; inoculação; Toona ciliata M. Roem var. australis

\section{ABSTRACT}

The Australian red cedar (Toona ciliata M. Roem var. Australis) is a species that has shown potential for forest use in Brazil. Thus, the objective of this study was to test the Australian red cedar seedlings response to inoculation with arbuscular mycorrhizal fungi (AMF) in different doses of $\mathrm{P}$. The experiment was conducted in a Completely Randomized Design (CRD) in factorial $8 \times 2$, with five repetitions. The treatments consisted in inoculation of seven species of AMF: Rhizophagus clarus, Claroideoglomus etunicatum, Racocetra gregaria, Acaulospora colombiana, Acaulospora morrowiae, Gigaspora margarita and Dentiscutata heterogama, besides a treatment without inoculation; being subjected to two doses of

1 Engenheira Agrônoma, Doutoranda no Programa de Pós-graduação em Recursos Genéticos Vegetais, Centro Ciências Agrárias, Universidade Federal de Santa Catarina, Rod. Admar Gonzaga, 1346 , Itacorubi, CEP 88034-000, Florianópolis (SC), Brasil.manu_pilles@hotmail.com

2 Engenheiro Agrônomo, Pós-doutorando no Programa de Pós-graduação em Ciência do Solo, Centro de Ciências Rurais, Universidade Federal de Santa Maria, Av. Roraima, 1000, CEP 97105-900, Santa Maria (RS), Brasil. ferreira.aap@gmail.com

3 Engenheiro Agrônomo, Dr. Pesquisador do Instituto Tecnológico Vale, Rua Boaventura da Silva, 415/416 a 1147/1148, Nazaré, CEP 66055-090, Belém (PA), Brasil. furtinineto@gmail.com

4 Engenheiro Agrônomo, Dr. Professor Adjunto do Departamento de Microbiologia Imunologia e Parasitologia, Universidade Federal de Santa Catarina, Campus Reitor João David Ferreira Lima, Caixa Postal 476, Trindade, CEP 88040-900, Florianópolis (SC), Brasil. crfsoares@gmail.com 
phosphate 25 and $250 \mathrm{mg} \mathrm{dm}^{-3}$ and that levels were equivalent to 10.7 and $41.3 \mathrm{mg} \mathrm{dm}^{-3}$, respectively. It was evaluated the percentage of colonization, sporulation, height, diameter, shoot dry matter and accumulation of nutrients, 150 days after transplanting. There were differences depending on the levels of $\mathrm{P}$ and isolated variables evaluated. At the lowest dose, the isolated Claroideoglomus etunicatum and Acaulospora colombiana benefit the growth and nutrient absorption, while at the highest dose, Acaulospora morrowiae showed to be more promising for the growth of plants. These isolates provided high mycorrhizal colonization in the red cedar, favoring the production of quality seedlings, aiming the establishment and productivity of commercial plantations.

Keywords: mycorrhizal association; phosphorus fertilization; forestry production; inoculation; Toona ciliata $\mathrm{M}$. Roem var. australis

\section{INTRODUÇÃO}

Plantios comerciais florestais assumem funções não apenas de produção, mas também de conservação do ambiente. Além de fornecerem matéria-prima para diferentes usos, contribuem para diversos serviços ambientais e sociais. Ademais, evitam a agressão aos recursos naturais por suprirem a crescente demanda por madeiras, reduzindo a necessidade da exploração das matas nativas (GARLIPP; FOELKEL, 2009).

Estudos silviculturais no Brasil têm se voltado quase que exclusivamente, para as espécies de rápido crescimento como, por exemplo, as do gênero Eucalyptus e Pinus. Isso pode ser observado pela área plantada com estas espécies que ocupam $70,8 \%$ e $22,0 \%$ do total da área de florestas plantadas, respectivamente, que equivalem a uma área plantada de pinus e eucalipto no território nacional de 6.664 .812 ha. A contribuição irrisória de outras espécies (não convencionais) no setor florestal brasileiro (7,2\%) (ASSOCIAÇÃO BRASILEIRA DE PRODUTORES DE FLORESTAS PLANTADAS, 2013), demonstra o grande potencial ainda inexplorado destas espécies. Deste modo, estudos devem ser realizados a fim de identificar espécies arbóreas promissoras, visando a uma maior diversificação da produção florestal por produtores e empresas.

Entre as espécies que têm se mostrado promissoras para o reflorestamento está o cedro-australiano (Toona ciliata M. Roem var. australis) (MORETTI et al., 2011). A espécie pertence à família Meliaceae, cuja origem se estende desde a Índia e Malásia até o norte da Austrália (LORENZI, 2003; PINHEIRO; LANI; COUTO, 2003). No Brasil, o cedro-australiano encontrou condições edafoclimáticas favoráveis ao seu desenvolvimento vegetativo e para a produção de madeira, sendo cultivado ao Sul do estado da Bahia e em toda a região Sudeste. No cenário nacional destacam-se os plantios em escala comercial nos estados do Espírito Santo e Minas Gerais, podendo apresentar incremento médio anual de $20 \mathrm{~m}^{3} \mathrm{ha}^{-1}$ ano $^{-1}$ (MURAKAMI, 2008).

Esta espécie possui similaridade botânica com os cedros nativos do Brasil do gênero Cedrella da Mata Atlântica e o mogno (Swietenia macrophylla King) da Floresta Amazônica (LORENZI 2003; PINHEIRO; LANI; COUTO, 2003), que são espécies produtoras de madeira com alto valor de mercado. Entretanto, o cultivo dessas plantas envolve a utilização de práticas agrícolas adequadas para a obtenção de povoamentos florestais produtivos. Esse processo tem início no estabelecimento de mudas de qualidade, as quais devem ser capazes de suportar as variações edafoclimáticas e, consequentemente, apresentar altos percentuais de sobrevivência no campo e menor necessidade de tratos culturais. Neste sentido, trabalhos têm sido desenvolvidos no intuito de melhorar a qualidade das mudas, bem como visando a redução dos custos de produção (PEZZUTTI; SCNUMACHER; HOPPE, 1999; SANTOS et al., 2000; GOMES et al., 2003).

A produção comercial de mudas de qualidade está normalmente vinculada ao fornecimento de nutrientes, especialmente de fósforo $(\mathrm{P})$, importante para o estabelecimento das culturas, uma vez que, em geral, o substrato utilizado possui fertilidade natural extremamente baixa (SCHUMACHER; CECONI; SANTANA, 2004). Apesar de o P ser exigido em baixas quantidades pelas plantas, há uma carência generalizada deste elemento nos solos brasileiros, devido a sua forte interação com outros componentes do solo (RAIJ, 1991). Moretti et al. (2011) verificaram por meio da técnica do elemento faltante, que mudas de

cedro-australiano são influenciadas significativamente pela falta de macronutrientes, sendo que a carência de $\mathrm{P}$ refletiu no menor crescimento das plantas, resultando em menor número de folhas, raiz principal mais longa, porém, com poucas raízes laterais. 
Entre as alternativas mais econômicas e sustentáveis para o fornecimento de nutrientes para a produção de mudas está o uso de microrganismos. Nos sistemas biológicos envolvendo planta-microrganismos, temse a simbiose mutualística entre certos fungos do solo e as raízes das plantas, denominada micorrizas. Dentre os vários tipos de micorrizas, destacam-se as micorrizas arbusculares que ocorrem na maioria das espécies florestais (MUTHUKUMAR, et al., 2003; SHI, et al. 2006; SOARES; CARNEIRO, 2010), incluindo o cedro-australiano (THAPAR; VIJYAN; UNIYAL, 1992). Esta simbiose aumenta a absorção de nutrientes, em especial o P, e a tolerância das plantas a diversos tipos de estresses, influenciando seu crescimento e reprodução (SIQUEIRA et al., 2007). Entretanto, os fungos micorrízicos arbusculares (FMA) apresentam eficiência simbiótica diferenciada para as espécies vegetais e, por esta razão, devem ser testados em uma mesma planta e sob as mesmas condições ambientais, visando selecionar isolados mais eficientes (SAGGIN JÚNIOR; SIQUEIRA, 1995).

Pesquisas relacionadas à inoculação de FMA em diferentes espécies arbóreas vêm sendo desenvolvidas a fim de se estabelecer a dependência desta interação, especialmente na formação das mudas. Carneiro et al. (1998) verificaram alta incidência de micorrizas (50\%) em Cedrella fissilis (cedro), baixa (1-19\%) em Melia azedarach (cinamomo) e média (20-49\%) em Carapa guianensis (andiroba), em condições de viveiro. Já Muthukumar et al. (2003) verificaram que a porcentagem de colonização radicular em Azadirachta indica (nim-indiano) foi muito elevada (70\%), enquanto que para Melia azedarach foi moderada (30\%). Tótola e Borges (2000) também observaram um efeito positivo da inoculação de FMA sobre o crescimento das plantas de Cedrella fissilis e um aumento na capacidade de absorção de P. Este resultado corrobora aqueles obtidos por Rocha et al. (2006) que verificaram um elevado grau de dependência micorrízica das mudas de cedro, demonstrando que a inoculação de FMA é um procedimento que deve ser considerado na fase de viveiro para garantir o sucesso do estabelecimento e crescimento dessas mudas em campo.

Deste modo, o objetivo do presente trabalho foi avaliar o efeito da inoculação de isolados de FMA, associada à aplicação de diferentes doses de $\mathrm{P}$, no crescimento e nutrição mineral do cedro-australiano na fase de produção de mudas.

\section{MATERIAL E MÉTODOS}

O ensaio foi realizado em casa de vegetação do Departamento de Ciência do Solo (DCS) da Universidade Federal de Lavras - UFLA, durante um período de 150 dias (julho a novembro de 2009). Foi coletado um Latossolo Vermelho distrófico (LVd), na cidade de Itutinga - MG, contendo 75\% de areia; 6\% de silte e $19 \%$ de argila. Esse solo possuía baixa fertilidade, apresentando os seguintes atributos químicos: $\mathrm{pH}_{\mathrm{H} 2 \mathrm{O}}=4,7 ; \mathrm{P}=0,6 \mathrm{mg} \mathrm{dm}^{-3}$ (Mehlich I); $\mathrm{K}=28 \mathrm{mg} \mathrm{dm}^{-3} ; \mathrm{Ca}=0,1 \mathrm{cmol}_{\mathrm{c}} \mathrm{dm}^{-3} ; \mathrm{Mg}=0,1 \mathrm{cmol}_{\mathrm{c}} \mathrm{dm}^{-3}$; $\mathrm{Al}=0,9 \mathrm{cmol}_{\mathrm{c}} \mathrm{dm}^{-3} ; \mathrm{H}+\mathrm{Al}=8,8 \mathrm{cmol}_{\mathrm{c}} \mathrm{dm}^{-3} ;$ e $\mathrm{MO}=3,0 \mathrm{dag} \mathrm{kg}^{-1}$.

Ao solo coletado foi adicionado calcário dolomítico para elevar a saturação por bases para $65 \%$ (RAIJ, 1991). Realizou-se a adubação de plantio $\left(300 \mathrm{mg} \mathrm{dm}^{-3}\right.$ de $\mathrm{N}$ na forma de $\mathrm{NH}_{4} \mathrm{NO}_{3} ; 40 \mathrm{mg} \mathrm{dm}{ }^{-3}$ de $\mathrm{S}$ na forma de $\mathrm{K}_{2} \mathrm{SO}_{4} ; 300 \mathrm{mg} \mathrm{dm}^{-3}$ de $\mathrm{K}$ na forma de $\mathrm{KCl}$ ), além da aplicação de micronutrientes $\left(1,5 \mathrm{mg} \mathrm{dm}^{-3} \mathrm{Cu}\right.$ na forma de $\mathrm{CuSO}_{4} \cdot 5 \mathrm{H}_{2} \mathrm{O} ; 5 \mathrm{mg} \mathrm{dm}^{-3} \mathrm{Zn}$ na forma de $\mathrm{ZnSO}_{4} \cdot 7 \mathrm{H}_{2} \mathrm{O} ; 0,8 \mathrm{mg} \mathrm{dm}^{-3} \mathrm{~B}$ na forma de $\mathrm{H}_{3} \mathrm{BO}_{3}$ e $0,15 \mathrm{mg} \mathrm{dm}^{-3}$ Mo na forma de $\left.\left(\mathrm{NH}_{4}\right) 6 \mathrm{Mo}_{7} \mathrm{O}_{24} \cdot \mathrm{H}_{2} \mathrm{O}\right)$.

Após esta etapa, o solo foi fumigado utilizando-se Brometo de Metila 98\% + Cloropicrina 2\% na dosagem de $196 \mathrm{~cm}^{3} \mathrm{~m}^{-3}$ de solo, a fim de eliminar propágulos de FMA nativos. Esse produto não é mais comercializado no Brasil, mas foi empregado durante a pesquisa pela sua disponibilidade no laboratório no momento da implantação do experimento. Para a instalação deste ensaio foram utilizados vasos com capacidade para $3,0 \mathrm{dm}^{3}$ de solo. $\mathrm{O}$ experimento foi conduzido em delineamento inteiramente casualizado (DIC) em esquema fatorial $8 \times 2$, com 5 repetições. Os tratamentos consistiram na inoculação de sete espécies de FMA: Rhizophagus clarus (Nicolson \& N.C. Schenck), Claroideoglomus etunicatum (W.N. Becker \& Gerd.), Racocetra gregaria (N.C. Schenck \& T.H. Nicolson), Acaulospora colombiana (Spain \& N.C. Schenck), Acaulospora morrowiae (Spain \& Schenck), Gigaspora margarita (Becker \& Hall) e Dentiscutata heterogama (Nicolson \& Gerd.), além de um tratamento sem inoculação. Todos esses tratamentos foram combinados com duas doses de fosfato $\left(25\right.$ e $\left.250 \mathrm{mg} \mathrm{dm}^{-3} \mathrm{P}\right)$ por meio da aplicação de superfosfato triplo $\left(41 \%\right.$ de $\left.\mathrm{P}_{2} \mathrm{O}_{5}\right)$. Os isolados foram obtidos da coleção de FMA do Laboratório de 
Microbiologia e Bioquímica do Solo da UFLA. Após 30 dias de incubação do solo com o adubo fosfatado, avaliaram-se as amostras de solo que apresentaram 10,7 e 41,3 $\mathrm{mg} \mathrm{dm}^{-3}$ de $\mathrm{P}$ disponível pelo extrator Mehlich-1, respectivamente. Sementes de cedro-australiano (Toona ciliata M. Roem var. australis) provenientes da BV Florestal, foram semeadas em bandejas com areia estéril até que as plântulas atingissem aproximadamente $10 \mathrm{~cm}$ de altura, sendo então realizado o transplantio, acondicionando-se quatro plântulas por vaso. Logo após o transplantio foi realizada a inoculação das mesmas por meio da aplicação de uma suspensão de esporos, de modo a fornecer 200 esporos de FMA por planta. Após 15 dias foi realizado desbaste deixando-se apenas uma planta por vaso.

Cinco meses após o transplantio, foram avaliados altura da planta, diâmetro do caule, produção de massa seca da parte aérea, porcentagem de colonização micorrízica, número de esporos e conteúdo de nutrientes na parte aérea. Calculou-se a cada 30 dias até o final do experimento, a taxa de crescimento relativa/mês (TCR/mês) pela equação $\left(\pi \cdot r^{2} . h\right) / I(S I E V E R D I N G, 1991)$, em que: $r=$ raio do caule; $h=$ altura das plantas e I = idade das plantas.

Ao final do experimento, as plantas foram cortadas ao nível do solo e a parte aérea foi lavada com água destilada, submetida à secagem em estufa à $60^{\circ} \mathrm{C}$, pesada e utilizada para análises de nutrientes. As raízes foram lavadas sobre peneira em água corrente e cerca de $1,0 \mathrm{~g}$ foi conservada em FAA [formaldeído (40\%): álcool (50\%): ácido acético $=13 \mathrm{~mL}: 200 \mathrm{~mL}: 5 \mathrm{~mL}$ ], para posterior clareamento e coloração pelo método de Phillips e Hayman (1970) e avaliação em placa quadriculada (GIOVANETTI; MOSSE, 1980). A eficiência micorrízica (EM) de cada isolado de FMA foi calculada com base no incremento da produção de massa seca da parte aérea da planta, conforme a equação: $\mathrm{EM}=[$ (peso de massa seca da planta inoculada - peso de massa seca da planta não inoculada)/peso de massa seca da planta não inoculada] x 100 (MOREIRA; SIQUEIRA, 2006). Esse valor indica se houve aumento (valor positivo) ou redução (valor negativo) da produção de massa seca das plantas inoculadas em comparação àquelas não-inoculadas (controle).

Os esporos foram extraídos pelo método de peneiragem úmida (GERDEMANN; NICOLSON, 1963), e posteriormente, por centrifugação em água a $2.000 \mathrm{rpm}$ durante três minutos e em sacarose (45\%) a $1.500 \mathrm{rpm}$ por dois minutos. A contagem foi feita com o auxílio de microscópio estereoscópico (40x). Após a determinação da massa seca da parte aérea, as amostras foram moídas e utilizadas para determinação do teor de nutrientes. O N foi determinado pelo método semimicro Kjeldahl, e a destilação e titulação, segundo Bremner e Edward (1965). No extrato obtido por digestão nítrico-perclórica, determinaram-se os teores de $\mathrm{Ca}, \mathrm{Mg}, \mathrm{S}, \mathrm{Mn}, \mathrm{Cu}$ e $\mathrm{Zn}$ por espectrofotometria de absorção atômica e de $\mathrm{P}$ por colorimetria.

Os dados foram submetidos à análise de variância e, quando significativo, as médias comparadas pelo teste Scott-Knott a 5\% de probabilidade. Os dados de colonização micorrízica e esporulação foram transformados em arcsen $(\mathrm{x} / 100)^{1 / 2}$ e $\log 10(\mathrm{x}+1)$, respectivamente, pelo programa estatístico SISVAR (FERREIRA, 2011). O coeficiente de correlação de Pearson $(\mathrm{P}<0,05)$ foi utilizado para avaliar a correlação entre as variáveis de colonização micorrízica, nutrição mineral e produção de biomassa das plantas, utilizando-se o programa SigmaPlot 12.0 (Systat Software, Inc.).

\section{RESULTADOS E DISCUSSÃO}

\section{Esporulação e associação micorrízica}

A esporulação e a colonização micorrízica foram significativamente influenciadas pela adição de fosfato e pela inoculação com FMA, sendo observada interação entre os fatores (doses de P x inoculação com FMA).

De maneira geral, verificou-se redução na esporulação dos FMA quando as mudas de cedro foram cultivadas na maior dose de $\mathrm{P}\left(250 \mathrm{mg} \mathrm{dm}^{-3}\right)$. Os tratamentos inoculados apresentaram esporulação variando de 15 a 32 esporos $50 \mathrm{~cm}^{3}$ solo $^{-1}$ na dose de $25 \mathrm{mg} \mathrm{dm}^{-3} \mathrm{P}$, enquanto que na dose de $250 \mathrm{mg} \mathrm{dm}^{-3}$ esta variou de 13 a 27 esporos $50 \mathrm{~cm}^{3}$ solo $^{-1}$. Na dose de $25 \mathrm{mg} \mathrm{dm}^{-3}$ de $\mathrm{P}$, os isolados Acaulospora colombiana e Claroideoglomus etunicatum apresentaram maior esporulação em relação aos demais isolados (Figura 1a). A aplicação de $250 \mathrm{mg} \mathrm{dm}^{-3} \mathrm{P}$ diminuiu significativamente a esporulação de Claroideoglomus etunicatum. Apesar disso, esse isolado de FMA foi, juntamente com Acaulospora colombiana, Dentiscutata heterogama 
e Rhizophagus clarus, o que apresentou maior esporulação nesta dose de P (Figura 1a).

Em relação à colonização micorrízica, pode-se observar que esta variou entre 10 e $48 \%$, na dose de $25 \mathrm{mg} \mathrm{dm}^{-3} \mathrm{P}$, enquanto que na dose de $250 \mathrm{mg} \mathrm{dm}^{-3}$ a variação foi de 35 e $60 \%$. No tratamento controle (sem inoculação), não foi constatada colonização micorrízica, indicando que não houve contaminação durante o experimento (Figura 1b). As mudas de cedro-australiano apresentaram os maiores valores de colonização micorrízica quando foram inoculadas com os isolados Acaulospora colombiana e Claroideoglomus etunicatum na dose de $25 \mathrm{mg} \mathrm{dm}^{-3}$.

Apesar de Acaulospora colombiana e Claroideoglomus etunicatum manterem a colonização micorrízica do cedro-australiano quando submetidas a doses mais elevadas de $\mathrm{P}$, os isolados Acaulospora morrowiae e Rhizophagus clarus apresentaram maior capacidade de colonizar as raízes das plantas na dose de $250 \mathrm{mg} \mathrm{dm}^{-3}$ (Figura 1b). Entre os isolados de FMA testados, verificou-se uma menor colonização micorrízica pelo isolado Racocetra gregaria, com percentagem de colonização inferior a $15 \%$ sob baixo P. São muito conhecidos os efeitos da disponibilidade de P sobre a colonização micorrízica e sabe-se que esse nutriente não torna as plantas imunes à colonização, reduzindo apenas a intensidade da micorrização.

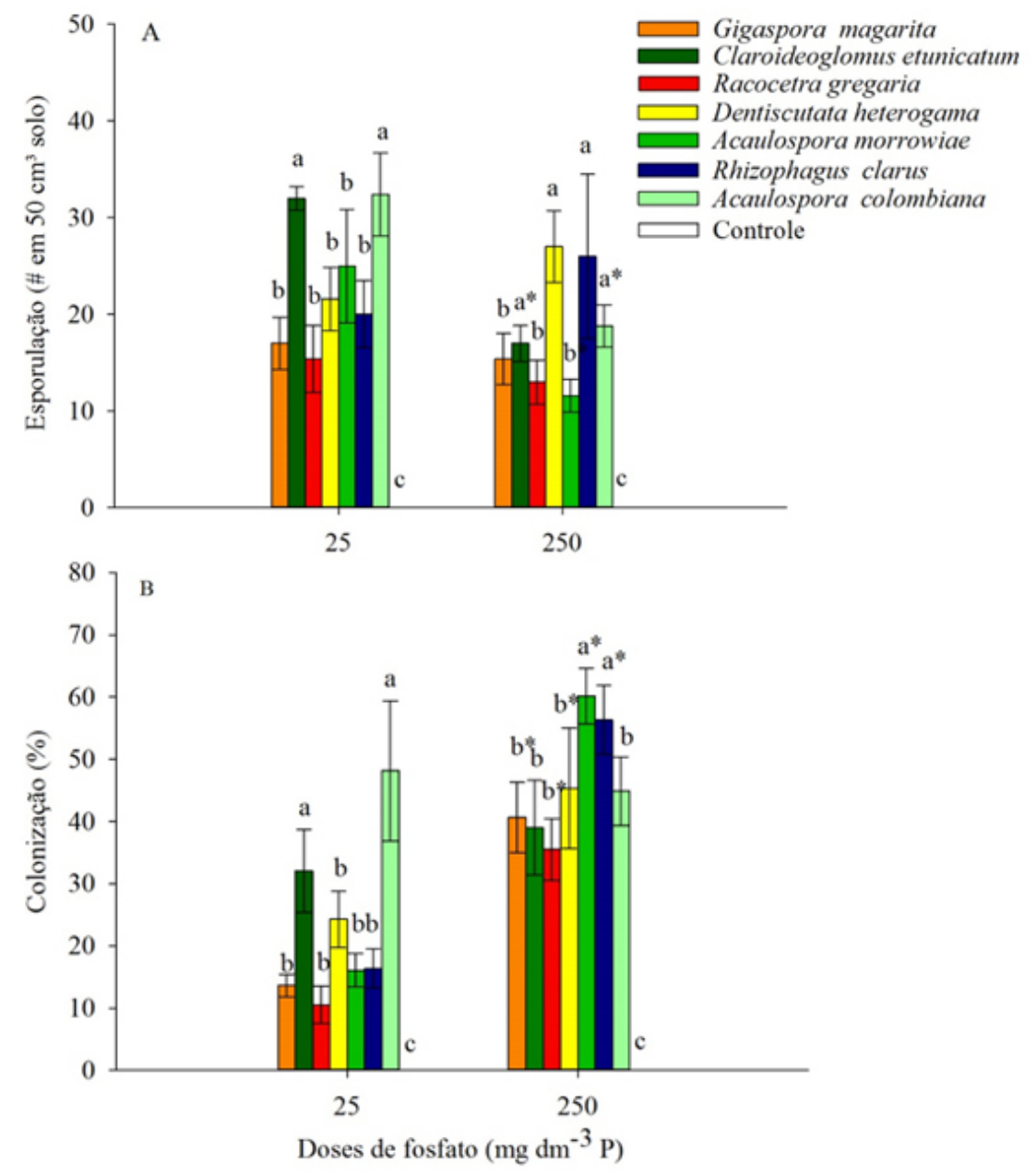

FIGURA 1: Número de esporos (A) e colonização micorrízica (B) em mudas de cedro-australiano inoculadas com diferentes fungos micorrízicos arbusculares aos 150 dias, sob aplicação de 25 e $250 \mathrm{mg}$ $\mathrm{dm}^{-3} \mathrm{P}$. Médias seguidas de mesma letra nas colunas (Doses de P) indicam um mesmo grupo pelo teste de Scott-Knott a 5\% de probabilidade. * Diferença significativa entre as doses de P.

FIGURE 1: Number of spores (A) and mycorrhizal colonization (B) of australian red cedar seedlings at 150 days inoculated with different arbuscular mycorrhizal fungi, treated with 25 and $250 \mathrm{mg} \mathrm{dm}^{-3}$ P. Means followed by same letter in columns (doses P) indicate the same group by Scott-Knott test at 5\% probability. ${ }^{*}$ Significant difference between the doses of $\mathrm{P}$. 
Entretanto, as condições edafoclimáticas e os mecanismos envolvidos são complexos e podem diferir entre as combinações fungo-planta da mesma espécie, dificultando generalizações sobre a associação micorrízica (MOREIRA; SIQUEIRA, 2006).

Shi et al. (2006), buscando compreender melhor a relação de FMA com espécies arbóreas da família Meliaceae na ilha tropical de Hainan (China) sob diferentes níveis de fertilidade natural de P, verificaram que os FMA colonizaram todas as 28 espécies de plantas analisadas. A porcentagem média geral de colonização verificada pelos autores foi de 56\%, variando entre 10 e $95 \%$. Os gêneros Acaulospora e Glomus foram dominantes, com $82 \%$ dos FMA identificados pertencentes a estes dois gêneros. Em contraste, os gêneros Entrophospora, Gigaspora e Scutellospora representaram apenas 3, 6, e 9\% do número de espécies, respectivamente. Alguns destes gêneros de FMA também foram encontrados nesta família em distintos estágios de desenvolvimento em ambientes florestais, conforme demonstrado por Rodríguez-Morelos et al. (2014). Os autores, estudando um fragmento de floresta tropical com mudas e árvores de $S$. macrophylla estabelecidas naturalmente, encontraram um total de 23 morfotipos de FMA. No solo da rizosfera de mudas, 11 morfotipos foram registrados, correspondentes aos gêneros Glomus e Acaulospora; enquanto na rizosfera do solo de árvores em campo, 21 morfotipos foram registrados em quatro gêneros (Glomus, Acaulospora, Gigaspora e Ambispora). Apenas nove morfotipos (Acaulospora scrobiculata, Acaulospora spinosa, Acaulospora sp. 2, Acaulospora sp. 5, Acaulospora sp. 6, Diversispora aurantium, Claroideoglomus etunicatum, Rhizophagus fasciculatus e Glomus sp. 3) foram comuns em ambas as fases de plantas. Deste modo, pode-se observar que em ambientes naturais há predominância dos dois gêneros aqui também estudados, Acaulospora e Glomus, os quais se destacaram quanto à colonização e esporulação. Isto demonstra que a associação com esses fungos poderia auxiliar as mudas de cedroaustraliano a sobreviverem após o transplante.

Os resultados do presente trabalho evidenciam que mudas de cedro-australiano apresentam elevada colonização micorrízica mesmo na maior dose de $\mathrm{P}$ aplicada. A adição de $25 \mathrm{mg} \mathrm{dm}^{-3} \mathrm{de} \mathrm{P}$, resultou em $10,7 \mathrm{mg} \mathrm{dm}^{-3}$ de $\mathrm{P}$ disponível, quantidade considerada baixa devido à textura do solo, de acordo com as Recomendações para o Uso de Corretivos e Fertilizantes para o Estado de Minas Gerais (COMISSÃO DE FERTILIZANTE DO SOLO DO ESTADO DE MINAS GERAIS, 1989). Já a adição de $250 \mathrm{mg} \mathrm{dm}^{-3}$ $\mathrm{P}$, proporcionou valores de $41,3 \mathrm{mg} \mathrm{dm}^{-3}$ de $\mathrm{P}$ disponível, quantidade essa considerada alta para esse tipo de solo. Desta forma, a adubação fosfatada combinada com a inoculação de FMA selecionados, pode favorecer o estabelecimento desta espécie arbórea em solos de baixa fertilidade, uma vez que as micorrizas arbusculares apresentam papel biofertilizador nas espécies vegetais com contribuições significativas na redução de estresse bióticos e abióticos (SOARES; CARNEIRO, 2010).

\section{Crescimento e produção de massa seca da parte aérea}

Em relação às variáveis morfológicas (altura e diâmetro do colo) e produção de massa seca da parte aérea (MSPA) foram observadas interações entre os fatores (níveis de P x inoculação com FMA).

Os FMA apresentaram comportamentos diferenciados sobre o crescimento das mudas de cedro-australiano (altura e diâmetro do caule) quando submetidos à dose mais baixa de P (Figura 2 a,b). Em relação à altura das plantas, a inoculação com os isolados Claroideoglomus etunicatum e Acaulospora colombiana se destacou dos demais tratamentos, proporcionando incrementos de $453 \%$ e $292 \%$, respectivamente, na altura das plantas em relação ao controle sem inoculação. Esses isolados, além de favorecerem o melhor crescimento das plantas em baixo $\mathrm{P}$, proporcionaram também um maior diâmetro do caule destas plantas, resultando em incrementos de 317 e $236 \%$, respectivamente, em relação às plantas não inoculadas. Estes resultados evidenciam os benefícios da inoculação de Claroideoglomus etunicatum e Acaulospora colombiana no vigor das mudas de cedro-australiano quando produzidas sob baixa disponibilidade de P. Entretanto, quando as mudas foram inoculadas sob alta dose de P, não foi observado o mesmo benefício dos FMA. Verifica-se que a inoculação não superou o crescimento das mudas quando submetidas a altas doses de $\mathrm{P}$, não havendo, portanto, diferenças na altura e diâmetro do caule das plantas quando do uso dos isolados de FMA e o tratamento não inoculado. Rocha et al. (2006), trabalhando com cedro (Cedrella fissilis) sob diferentes doses, verificaram que houve benefícios da inoculação de Rhizophagus clarus e Gigaspora margarita na altura das mudas aos 180 DAE, enquanto que, para o 

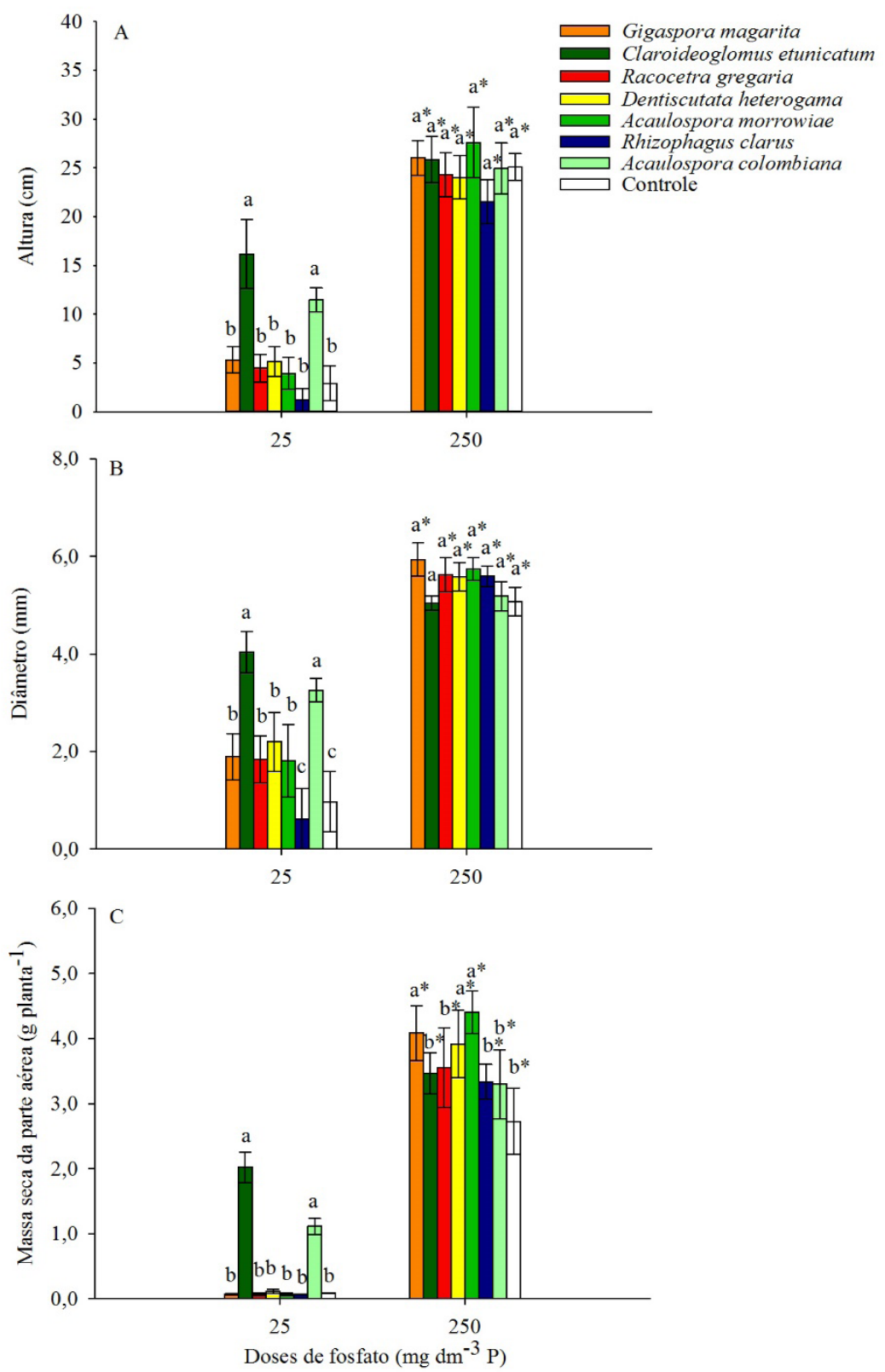

FIGURA 2: Efeito da inoculação de FMA (A) no crescimento (altura), (B) diâmetro do colo (C) matéria seca da parte áerea (MSPA) do cedro-australiano aos 150 dias, sob aplicação de 25 e $250 \mathrm{mg}$ $\mathrm{dm}^{-3}$ de P. Médias seguidas pela mesma letra não diferem entre si pelo teste Scott-Knott a $5 \%$ de probabilidade. * Diferença significativa entre as doses de $\mathrm{P}$.

FIGURE 2: Effect of AMF inoculation (A) on the growth (height), (B) stem diameter, (C), shoot dry matter (SDM) of Australian red cedar at 150 days, treated with 25 and $250 \mathrm{mg}$ P. Means followed by same letter do not differ by the Scott-Knott test at $5 \%$ probability. * Significant difference between the doses of $\mathrm{P}$.

diâmetro do caule, não houve efeito significativo da inoculação. Pouyu-Rojas, Siqueira e Santos (2006), ao trabalhar com 16 espécies florestais de distintos grupos sucessionais com inoculação de diferentes FMA, constataram diferenças entre os distintos FMA testados na colonização de mudas de espécies arbóreas e na produção de MSPA. Segundo Saggin Júnior e Siqueira (1995), a magnitude da eficiência simbiótica pode 
diferir entre os FMA testados e entre as variáveis consideradas.

Com relação à produção de massa seca da parte aérea, na dose de $25 \mathrm{mg} \mathrm{dm}^{-3} \mathrm{P}$, pode-se observar que os isolados que possuíram menor colonização micorrízica nesta dose, resultaram em plantas com menor produção de MSPA, não diferindo do tratamento sem inoculação. Entretanto, houve efeito positivo da inoculação de Claroideoglomus etunicatum e Acaulospora colombiana (Figura 2c), em relação ao tratamento não inoculado. Estes últimos obtiveram também maior taxa de colonização nesta dose. Já a aplicação de $250 \mathrm{mg} \mathrm{dm}^{-3} \mathrm{P}$ aumentou a produção de massa seca das mudas, com destaque para os tratamentos inoculados com Dentiscutata heterogama, Gigaspora margarita e Acaulospora morrowiae (Figura 2c). Verificou-se também que a MSPA das plantas se correlacionou positivamente com a taxa de colonização micorrízica na dose de $250 \mathrm{mg} \mathrm{dm}^{-3} \mathrm{de} \mathrm{P}(\mathrm{r}=0,78 ; \mathrm{P}<0,01)$. Já na dose de $25 \mathrm{mg} \mathrm{dm}^{-3}$ de $\mathrm{P}$, não se observou tal correlação. Isso provavelmente está relacionado com a baixa disponibilidade desse nutriente para o cedro-australiano, não atendendo de maneira satisfatória à demanda nutricional mesmo em plantas micorrizadas. Além disso, a capacidade do fungo em estimular o crescimento vegetal é determinada pelas características e por todos os componentes da simbiose, principalmente do microbionte que pode apresentar graus diferenciados de eficiência, sendo até mesmo ineficaz ou parasita temporário, dependendo da planta hospedeira e das condições de crescimento (SMITH; READ, 2008).

Deste modo, observam-se diferentes efeitos sobre as variáveis quando se avalia a interação entre a aplicação de fosfato e inoculação micorrízica. No caso de produção de mudas de espécies arbóreas que serão transplantadas a campo sob condições de estresse, a produção de MSPA pode ser uma variável que facilite e, portanto, influencie de forma positiva a sua sobrevivência à campo. Deste modo, atenção especial deve ser dada aos tratamentos que influenciem de forma mais significativa essa variável. Amplas faixas de P disponíveis são utilizadas em estudos envolvendo a inoculação de FMA em mudas de espécies arbóreas. Siqueira e Colozzi-Filho (1986), com base nos estudos com cafeeiro, estimaram que os teores de P no solo extraídos por Mehlich I, no qual se encontram as zonas de transição entre os estados parasítico-mutualista e mutualista-parasítico da simbiose micorrízica variam entre $1 \mathrm{e} 10 \mathrm{mg} \mathrm{dm}^{-3}$ e entre $100 \mathrm{e}$ $300 \mathrm{mg} \mathrm{dm}^{-3}$, respectivamente. Os autores estimaram esses valores para o cafeeiro, entretanto, as faixas podem ser válidas para várias espécies vegetais. Rocha et al. (2006), trabalhando com Cedrella fissilis, encontrou que o limite inferior da faixa de mutualismo, em termos de P disponível, ficou entre 2 e 14 $\mathrm{mg} \mathrm{dm}{ }^{-3}$ e o limite superior entre 124 e $144 \mathrm{mg} \mathrm{dm}^{-3}$, variando com a espécie de fungo e levando-se em consideração a MSPA. Já, quando é levada em consideração a altura das plantas, o limite inferior é de $2 \mathrm{mg}$ $\mathrm{dm}^{-3}$ e o superior varia entre $129-145 \mathrm{mg} \mathrm{dm}^{-3}$. Deste modo, pode-se observar que a dose de $25 \mathrm{mg} \mathrm{dm}^{-3}$, que corresponde a $10,7 \mathrm{mg} \mathrm{dm}^{-3}$ de $\mathrm{P}$ disponível no solo, encontra-se próxima do limite inferior da faixa de mutualismo, o que pode explicar a baixa contribuição de muitos isolados nesta dose. Assim sendo, os benefícios da aplicação conjunta da adubação fosfatada e de inoculantes micorrízicos devem ser avaliados em testes de eficiência empregando-se isolados de FMA benéficos para a espécie vegetal de interesse e dose de $\mathrm{P}$ recomendada.

Com base na massa seca da parte aérea, calculou-se a eficiência micorrízica de cada isolado fúngico, para se determinar a máxima resposta de cada FMA no crescimento das mudas de cedro (Tabela 1). Em condições de baixo P no solo $\left(25 \mathrm{mg} \mathrm{dm}^{-3}\right)$, os isolados Claroideoglomus etunicatum e Acaulospora colombiana apresentaram uma alta eficiência simbiótica, proporcionando incrementos de cerca de 2.248 e $1.190 \%$ na MSPA, respectivamente, em relação ao controle. Os benefícios dos isolados Claroideoglomus etunicatum e Acaulospora colombiana para o cedro-australiano podem estar relacionados com a sua maior eficiência em absorver nutrientes do solo e mantê-lo em altos níveis nos tecidos da planta (SOARES; CARNEIRO, 2010), conforme será discutido mais adiante:

Ainda na dose de $25 \mathrm{mg} \mathrm{dm}^{-3} \mathrm{P}$, os valores da eficiência micorrízica para os isolados Gigaspora margarita, Racocetra gregaria, Acaulospora morrowiae e Rhizophagus clarus foram muito baixos evidenciando o efeito negativo desses isolados na simbiose micorrízica para o cedro-australiano. Esse fato pode estar relacionado com a colonização do sistema radicular por estes isolados (Figura 1), os quais consumiriam maior quantidade de fotoassimilados (maior dreno de carbono), acarretando em um menor desenvolvimento da planta simbiôntica (SAGGIN JÚNIOR; SILVA, 2006) investindo assim, em colonização e esporulação e deixando a planta sem benefício recíproco.

A maior eficiência da inoculação na dose de $250 \mathrm{mg} \mathrm{dm}^{-3} \mathrm{P}$ foi observada para os isolados 
TABELA 1: Eficiência micorrízica e crescimento relativo de mudas de cedro-australiano, inoculadas com FMA e sob aplicação de diferentes doses de P aos 150 dias.

TABLE 1: Mycorrhizal efficiency and relative growth rate of Australian red cedar, inoculated with AMF and under different doses of $\mathrm{P}$ at 150 days.

\begin{tabular}{|c|c|c|c|c|}
\hline \multirow{3}{*}{ Tratamentos de inoculação de FMA } & \multicolumn{2}{|c|}{ Eficiência micorrízica } & \multicolumn{2}{|c|}{ Crescimento Relativo/Mês } \\
\hline & $25 \mathrm{mg} \mathrm{dm}^{-3} \mathrm{P}$ & $250 \mathrm{mg} \mathrm{dm}^{-3} \mathrm{P}$ & $25 \mathrm{mg} \mathrm{dm}^{-3} \mathrm{P}$ & $250 \mathrm{mg} \mathrm{dm}^{-3} \mathrm{P}$ \\
\hline & \multicolumn{2}{|c|}{ 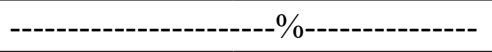 } & \multicolumn{2}{|c|}{-------------------------cm-------------- } \\
\hline Gigaspora margarita & $-16,28$ & 49,70 & 0,05 & 1,45 \\
\hline Claroideoglomus etunicatum & 2248,84 & 27,05 & 0,51 & 1,05 \\
\hline Racocetra gregaria & $-18,60$ & 30,13 & 0,04 & 1,26 \\
\hline Dentiscutata heterogama & 30,23 & 43,62 & 0,07 & 1,23 \\
\hline Acaulospora morrowiae & $-20,93$ & 61,51 & 0,06 & 1,50 \\
\hline Rhizophagus clarus & $-27,90$ & 22,28 & 0,02 & 1,08 \\
\hline Acaulospora colombiana & 1193,02 & 20,96 & 0,21 & 1,09 \\
\hline Controle & --- & --- & 0,03 & 1,05 \\
\hline
\end{tabular}

Acaulospora morrowiae e Gigaspora margarita, sendo esses recomendados para serem utilizados em solo com maior disponibilidade desse nutriente. Os benefícios da inoculação de FMA para o crescimento do cedro-australiano também podem ser constatados pela taxa de crescimento relativa ao mês, em que mudas inoculadas com Claroideoglomus etunicatum e Acaulospora colombiana apresentaram incrementos superiores a $600 \%$ em baixo P, enquanto Acaulospora morrowiae e Gigaspora margarita apresentaram maior taxa de crescimento relativa ao mês em alto $\mathrm{P}$, com incrementos médios de $40 \%$ em relação ao tratamento controle não inoculado. Deste modo, pode-se observar que há a necessidade de uma seleção de isolados de FMA mais específicos para o cedro-australiano em função da disponibilidade de P no solo visando à produção das mudas.

\section{Acúmulo de nutrientes}

Em relação ao acúmulo de nutrientes na parte aérea, exceto para o elemento $\mathrm{Mg}$, foram observadas interações entre os fatores (níveis de $\mathrm{P} x$ inoculação com FMA).

O conteúdo dos nutrientes nas mudas de cedro, de acordo com a dose de $\mathrm{P}$ aplicada e a inoculação dos FMA, pode ser observada na Tabela 2. Pode se verificar que na dose de $25 \mathrm{mg} \mathrm{dm}^{-3}$, as plantas inoculadas com os isolados Claroideoglomus etunicatum e Acaulospora colombiana apresentam maiores quantidades acumuladas de $\mathrm{P}, \mathrm{N}, \mathrm{Ca}, \mathrm{Mg}, \mathrm{S}, \mathrm{Cu}$ e $\mathrm{Zn}$, que os demais tratamentos. Somente o acúmulo de $\mathrm{Mn}$, não apresentou diferença significativa entre os tratamentos de inoculação. Os isolados Claroideoglomus etunicatum e Acaulospora colombiana proporcionaram aumento do conteúdo de P na ordem de $3.300 \%$ e $2.400 \%$ em relação ao controle, respectivamente, nessa dose. $\mathrm{O}$ acúmulo de $\mathrm{P}$ na parte aérea da planta correlacionou-se positivamente com a colonização micorrízica, tanto na dose de $25 \mathrm{mg} \mathrm{dm}-3 \mathrm{P}(\mathrm{r}=0,78$; $\mathrm{P}<0,01)$, como na dose de $250 \mathrm{mg} \mathrm{dm}-3 \mathrm{P}(\mathrm{r}=0,72 ; \mathrm{P}<0,01)$. Desta forma, considerando a eficiência simbiótica com base na produção de MSPA, verifica-se elevado grau de dependência micorrízica das plantas de cedroaustraliano. Em relação aos demais nutrientes, Claroideoglomus etunicatum e Acaulospora colombiana proporcionaram incrementos médios de $2.090 \%, 1.490 \%, 1.030 \%, 900 \%$ e $1.500 \%$ respectivamente, nos teores de $\mathrm{Ca}, \mathrm{Mg}, \mathrm{S}, \mathrm{Cu}$ e Zn. Correlações entre a colonização micorrízica e os teores de nutrientes foram observadas também em espécies florestais da Amazônia (OLIVEIRA; GUITTON; MOREIRA, 1999). Chen et al. (2005) também verificaram que Digitaria ciliares e Ixeris denticulate possuiram correlação positiva entre a taxa de infecção de raízes e concentração de $P$ na parte aérea.

Entretanto, apesar da correlação positiva entre colonização radicular e teor de $\mathrm{P}$ acumulado na parte aérea, a colonização pode não expressar diretamente o benefício micorrízico à planta, pois não reflete a 
capacidade de produção de micélio extrarradicular, que é responsável pelo aumento acentuado na absorção de P em plantas micorrizadas (BOLAN, 1991). Segundo o mesmo autor, o aumento na capacidade das plantas em absorver o $\mathrm{P}$ pode ser atribuído à capacidade dos fungos em aumentar, em mais de cem vezes, a área de exploração do solo pela grande produção de hifas extrarradiculares. Isso aumenta a eficiência na absorção de nutrientes das raízes quando na presença do fungo, uma vez que plantas micorrizadas têm acesso às mesmas formas de $\mathrm{P}$ disponíveis que as plantas não micorrizadas. Contudo, entre alguns fatores dos FMA que podem influenciar a absorção de P encontram-se a eficiência dos arbúsculos, a extensão, viabilidade e a capacidade de transporte da hifa externa. Além desses, a planta simbiôntica pode contribuir pela produção de pelos radiculares, o transporte e a utilização do $\mathrm{P}$ e a taxa de crescimento e a exigência nutricional (SMITH; READ, 2008).

$\mathrm{Na}$ dose de $250 \mathrm{mg} \mathrm{dm}^{-3}$ verificou-se um aumento na concentração de todos os elementos na parte aérea em relação à dose de $25 \mathrm{mg} \mathrm{dm}^{-3} \mathrm{e}$ isso se deve principalmente à maior produção de biomassa devido à adubação fosfatada. Na maior dose, os isolados mais eficientes em aumentar o conteúdo de $\mathrm{P}$ foram: Gigaspora margarita, Claroideoglomus etunicatum, Acaulospora morrowiae e Rhizophagus clarus (Tabela 2). Estes apresentaram incrementos de $33 \%, 40 \%, 51 \%$ e $33 \%$ em relação ao controle não inoculado, respectivamente. Todavia, dentre estes isolados, Acaulospora morrowiae merece atenção especial, pois

TABELA 2: Efeito da inoculação de fungos micorrízicos arbusculares (FMA) no conteúdo de macronutrientes e micronutrientes na parte aérea de mudas de cedro-australiano (Toona ciliata M. Roem var. australis) aos 150 dias, sob aplicação de 25 e $250 \mathrm{mg} \mathrm{dm}^{-3}$ de $\mathrm{P}$.

TABLE 2: Effect of arbuscular mycorrhiza fungi (AMF) inoculation in the contents of macro and micronutrients in the shoots of Australian red cedar seedlings (Toona ciliata M. Roem var. australis) at 150 days, treated with 25 and $250 \mathrm{mg} \mathrm{dm}^{-3}$ of $\mathrm{P}$.

\begin{tabular}{|c|c|c|c|c|c|c|c|c|c|}
\hline \multirow{2}{*}{ Nutrientes } & \multirow{2}{*}{ Doses de $\mathrm{P}$} & \multicolumn{8}{|c|}{ Tratamentos } \\
\hline & & Controle & $\mathrm{Gm}$ & $\mathrm{Ce}$ & $\mathrm{Rg}$ & $\mathrm{Dh}$ & $\mathrm{Am}$ & $\mathrm{Rc}$ & Ac \\
\hline mg planta ${ }^{-1}$ & $\mathrm{mg} \mathrm{dm}-3$ & & & & & & & & \\
\hline \multirow{2}{*}{$P$} & 25 & $0,06 \mathrm{~b}$ & $0,05 b$ & $2,04 a$ & $0,044 \mathrm{~b}$ & $0,11 b$ & $0,05 b$ & $0,05 b$ & $1,50 \mathrm{a}$ \\
\hline & 250 & $3,63 b^{*}$ & $4,82 a^{*}$ & $5,08 \mathrm{a}^{*}$ & $4,08 b^{*}$ & $4,03 b^{*}$ & $5,48 a^{*}$ & $4,82 a^{*}$ & $4,22 b^{*}$ \\
\hline \multirow{2}{*}{$\mathrm{N}$} & 25 & n.d. & n.d. & $6,95 \mathrm{a}$ & n.d. & n.d. & n.d. & n.d. & $4,21 \mathrm{a}$ \\
\hline & 250 & $10,41 a^{*}$ & $14,69 a^{*}$ & $11,84 a^{*}$ & $13,11 a^{*}$ & $14,29 a^{*}$ & $15,10 a^{*}$ & $11,84 a^{*}$ & $13,39 a^{*}$ \\
\hline \multirow{2}{*}{$\mathrm{Ca}$} & 25 & $1,05 b$ & $1,20 \mathrm{~b}$ & $25,73 a$ & $0,69 b$ & $1,64 b$ & $0,76 \mathrm{~b}$ & $0,55 \mathrm{~b}$ & $20,25 \mathrm{a}$ \\
\hline & 250 & $40,88 b^{*}$ & $70,59 a^{*}$ & $48,47 b^{*}$ & $50,59 b^{*}$ & $58,15 \mathrm{a}^{*}$ & $60,93 a^{*}$ & $54,11 b^{*}$ & $51,33 b^{*}$ \\
\hline \multirow{2}{*}{$\mathrm{Mg}$} & 25 & $0,51 \mathrm{~b}$ & $0,54 b$ & $8,80 \mathrm{a}$ & $0,37 \mathrm{~b}$ & $0,77 b$ & $0,38 b$ & $0,27 \mathrm{~b}$ & $7,26 \mathrm{a}$ \\
\hline & 250 & $13,23 \mathrm{a}^{*}$ & $17,06 a^{*}$ & $15,20 \mathrm{a}^{*}$ & $12,02 \mathrm{a}^{*}$ & $17,19 a^{*}$ & $16,74 a^{*}$ & $15,80 \mathrm{a}^{*}$ & $18,02 \mathrm{a}^{*}$ \\
\hline \multirow{2}{*}{ S } & 25 & $0,21 \mathrm{~b}$ & $0,36 \mathrm{~b}$ & $3,04 a$ & $0,12 b$ & $0,21 b$ & $0,11 \mathrm{~b}$ & $0,13 b$ & $1,76 \mathrm{a}$ \\
\hline & 250 & $5,13 \mathrm{a}^{*}$ & $6,71 a^{*}$ & $5,57 \mathrm{a}^{*}$ & $6,25 \mathrm{a}^{*}$ & $6,48 a^{*}$ & $7,68 \mathrm{a}^{*}$ & $5,72 \mathrm{a}^{*}$ & $6,16 a^{*}$ \\
\hline \multicolumn{10}{|l|}{$\mu \mathrm{g}$ planta $^{-1}$} \\
\hline \multirow{2}{*}{$\mathrm{Cu}$} & 25 & $0,92 \mathrm{~b}$ & $0,65 b$ & $10,40 \mathrm{a}$ & $1,09 b$ & $0,85 b$ & $0,45 b$ & $0,69 b$ & $7,87 \mathrm{a}$ \\
\hline & 250 & $10,92 b^{*}$ & $15,10 b^{*}$ & $14,12 \mathrm{~b}$ & $12,22 b^{*}$ & $13,02 b^{*}$ & $20,83 a^{*}$ & $12,74 b^{*}$ & $11,52 \mathrm{~b}$ \\
\hline \multirow{2}{*}{$\mathrm{Mn}$} & 25 & $11,71 \mathrm{a}$ & $12,85 \mathrm{a}$ & $91,84 \mathrm{a}$ & $7,92 \mathrm{a}$ & $17,11 \mathrm{a}$ & $8,66 a$ & $6,77 \mathrm{a}$ & $66,27 \mathrm{a}$ \\
\hline & 250 & $186,19 b^{*}$ & $342,15 a^{*}$ & $195,86 b^{*}$ & $262,97 a^{*}$ & $294,11 a^{*}$ & $277,48 a^{*}$ & $276,52 a^{*}$ & $256,08 a^{*}$ \\
\hline \multirow{2}{*}{$\mathrm{Zn}$} & 25 & $1,67 \mathrm{c}$ & $0,38 \mathrm{c}$ & $33,86 a$ & $1,57 \mathrm{c}$ & $2,49 \mathrm{c}$ & $1,62 \mathrm{c}$ & $1,44 \mathrm{c}$ & $19,52 b$ \\
\hline & 250 & $35,55 b^{*}$ & $47,44 b^{*}$ & $42,90 \mathrm{~b}$ & $35,46 b^{*}$ & $46,44 b^{*}$ & $73,89 a^{*}$ & $35,35 b^{*}$ & $36,89 b^{*}$ \\
\hline
\end{tabular}

Em que: n.d. = não determinado; Gm: Gigaspora margarita; Ce: Claroideoglomus etunicatum; Rg: Racocetra gregaria; Dh: Dentiscutata heterogama; Am: Acaulospora morrowiae; Rc: Rhizophagus clarus; Ac: Acaulospora colombiana. Médias seguidas de mesma letra, minúsculas nas linhas (inoculação) não diferem entre si pelo teste de Scott-Knott a $5 \%$ de probabilidade. * Diferença significativa entre as doses de P. 
proporcionou absorção elevada não somente de $\mathrm{P}$, mas também de outros elementos, como $\mathrm{Ca}, \mathrm{Cu}, \mathrm{Mn} \mathrm{e}$ Zn, apresentando aumentos de $49 \%, 91 \%$, 49\% e 106\%, respectivamente.

Deste modo, pode-se observar que há um sinergismo entre as doses de fosfato aplicadas e a inoculação micorrízica, com relação ao acúmulo de nutrientes em mudas de cedro-australiano. Na dose mais baixa de $\mathrm{P}$, os isolados Claroideoglomus etunicatum e Acaulospora colombiana foram capazes de beneficiar as plantas. Por outro lado, na maior dose de $\mathrm{P}$, o isolado Acaulospora morrowiae proporcionoumaior acúmulo de nutrientes.

Entretanto, estudos adicionais devem ser realizados com doses graduais de adubação fosfata e FMA aqui testados, de modo a permitir a obtenção de curvas de adubação fosfatada que possam predizer em qual quantidade e com quais isolados fúngicos são obtidas as melhores respostas para a produção de mudas de cedro-australiano.

\section{CONCLUSÕES}

O crescimento do cedro-australiano (Toona ciliata M. Roem var. australis) é favorecido pela inoculação de fungos micorrízicos arbusculares (FMA) em solos de baixa fertilidade natural

Os fungos micorrízicos Claroideoglomus etunicatum e Acaulospora colombiana apresentam elevada colonização micorrízica com o cedro-australiano e beneficiam o crescimento e a absorção de $\mathrm{P}, \mathrm{N}$, $\mathrm{Ca}, \mathrm{Mg}, \mathrm{S}, \mathrm{Cu}$ e $\mathrm{Zn}$ em solo contendo baixa disponibilidade de $\mathrm{P}$.

A inoculação de Acaulospora morrowiae proporciona elevada eficiência micorrízica e aumento na absorção de nutrientes para mudas de cedro-australiano em solo com alta disponibilidade de $\mathrm{P}$.

\section{REFERÊNCIAS}

ASSOCIAÇÃO BRASILEIRA DE PRODUTORES DE FLORESTAS PLANTADAS. Anuário estatístico 2013, ano base 2012. Brasília: ABRAF, 2013. 148 p.

BOLAN, N. S. A critical review on the role of mycorrhizal fungi in the uptake of phosphorus by plants. Plant and Soil, Netherlands, v. 134, p. 189-207, 1991.

BREMNER, J. M.; EDWARDS, H. P. Determination and isotope ratio analysis of different forms of nitrogen in soils. I. Apparatus and procedures for distillation and determination for ammonium. Soil Science Society of America Proceedings, Madison, v. 29, n. 5, p. 504-507, sept. 1965.

CARNEIRO, M. A. C. et al. Micorriza arbuscular em espécies arbóreas e arbustivas nativas de ocorrência no sudeste do Brasil. Cerne, Lavras, v. 4, n. 1, p. 129-145, 1998.

CHEN, X. et al. Arbuscular mycorrhizal colonization and phosphorus acquisition of plants: effects of coexisting plant species. Applied Soil Ecology, Amsterdam, v. 28, p. 259-269, 2005.

COMISSÃO DE FERTILIZANTE DO SOLO DO ESTADO DE MINAS GERAIS. Recomendações para o uso de corretivos e fertilizantes em Minas Gerais: 4ª aproximação. Lavras: CFSEMG, 1989. 159 p.

FERREIRA, D. F. Sisvar: a computer statistical analysis system. Ciência e Agrotecnologia, Lavras, v. 35, n. 6, p. 1039-1042, nov./dez. 2011.

GARLIPP, R.; FOELKEL C. O papel das florestas plantadas para atendimento das demandas futuras da sociedade. In: WORLD FORESTRY CONGRESS, 13., Buenos Aires. Anais... [s.l.]: Sociedade Brasileira de Silvicultura, 2009. 18 p.

GERDEMANN, J. B.; NICOLSON, T. H. Spores of mycorrhizal Endogone species extracted from soil by wet sieving and decanting. Transactions of the British Mycological Society, London, v. 46, n. 2, p. 235-244, 1963.

GIOVANNETTI, M.; MOSSE, B. An evaluation of techniques for measuring vesicular arbuscular mycorrhizal infection in roots. New Phytologist, Cambridge, v. 84, n. 3, p. 489-500, 1980.

GOMES, J. M. et al. Crescimento de mudas de Eucalyptus grandis em diferentes tamanhos de tubetes e fertilização N-P-K. Revista Árvore, Viçosa, MG, v. 27, n. 2, abr. 2003.

LORENZI, H. Árvores exóticas no Brasil: madeireiras, ornamentais e aromáticas. Nova Odessa: Instituto Plantarum da Flora, 2003. 368 p.

MOREIRA, F. M. S.; SIQUEIRA, J. O. Microbiologia e bioquímica do solo. Lavras: Editora UFLA, 2006. 
$729 \mathrm{p}$.

MORETTI, B. S. et al. Crescimento e nutrição mineral de mudas de cedro australiano (Toona ciliata) sob omissão de nutrientes. Cerne, Lavras, v. 17, n. 4, p. 453-463, out./dez. 2011.

MURAKAMI, C. H. G. Cedro australiano: valorização de espécies nobres. Boletim Florestal, São Paulo, v. 7, n. 2, p. 1-6, fev. 2008.

MUTHUKUMAR, T. et al. Mycorrhiza of plants in different vegetation types in tropical ecosystems of Xishuangbanna, southwest China. Mycorrhiza, New York, v. 13, n. 6, p. 289-297, dec. 2003.

OLIVEIRA, L. A.; GUITTON, T. L.; MOREIRA, F. W. Relações entre as colonizações por fungos micorrízicos arbusculares e teores de nutrientes foliares em oito espécies florestais da Amazônia. Acta Amazonica, Manaus v. 29, n. 2, p. 183-193, 1999.

PEZZUTTI, R. V.; SCNUMACHER, M. V.; HOPPE, J. M. Crescimento de mudas de Eucalyptus globulus em resposta à fertilização NPK. Ciência Florestal, Santa Maria, v. 9, n. 2, p. 117-125, 1999.

PHILLIPS, J. M.; HAYMANN, D. S. Improved procedures for clearing roots and staining parasitic and vesicular-arbuscular mycorrhizal fungi for rapid assessment of infection. Transactions British Mycological Society, London, v. 55, n. 1, p. 158-161, 1970.

PINHEIRO, A. L.; LANI, L. L.; COUTO, L. Cultura do cedro australiano para produção de madeira serrada. Viçosa, MG: Editora UFV, 2003. 42 p.

POUYU-ROJAS, E.; SIQUEIRA, J. O.; SANTOS, J. G. D. Compatibilidade simbiótica de fungos micorrízicos arbusculares com espécies arbóreas tropicais. Revista Brasileira de Ciência do Solo, Viçosa, MG, v. 30, n. 3, jun. 2006.

RAIJ, B. van. Fertilidade do solo e adubação. Piracicaba: Agronômica Ceres; Associação Brasileira para a Pesquisa da Potassa e do Fosfato, 1991. 343 p.

ROCHA, F. S. et al. Dependência e resposta de mudas de cedro a fungos micorrízicos arbusculares. Pesquisa Agropecuária Brasileira, Brasília v. 41, n. 1, p. 77-84, jan. 2006.

RODRÍGUEZ-MORELOS, V. H. et al. Arbuscular mycorrhizal fungi associated with the rhizosphere of seedlings and mature trees of Swietenia macrophylla (Magnoliophyta: Meliaceae) in Los Tuxtlas, Veracruz, Mexico. Revista Chilena de Historia Natural, Santiago, v. 87, n. 9, p. 1-10, 2014.

SAGGIN JÚNIOR, O. J.; SILVA, E. M. R. Micorriza arbuscular: papel, funcionamento e aplicação da simbiose. Seropédica: Embrapa Agrobiologia, 2006. p. 101-149.

SAGGIN JÚNIOR, O. J.; SIQUEIRA, J. O. Avaliação da eficiência simbiótica de fungos endomicorrízicos no cafeeiro. Revista Brasileira de Ciência do Solo, Campinas, v. 19, p. 221-228, 1995.

SANTOS, C. B. et al. Efeito do volume de tubetes e tipos de substratos na qualidade de mudas de Cryptomeria japonica (L.F.) D. Don. Ciência Florestal, Santa Maria, v. 10, n. 2, p. 1-15, 2000.

SCHUMACHER, M. V.; CECONI, D. E.; SANTANA, C. A. Influência de diferentes doses de fósforo no crescimento de mudas de angico-vermelho (Parapiptadenia rigida (Bentham) Brenan). Revista Árvore, Viçosa, MG, v. 28, n. 1, p. 149-155, 2004.

SHI, Z. Y. et al. Arbuscular mycorrhizal fungi associated with the Meliaceae on Hainan island, China. Mycorrhiza, New York, v. 16, p. 81-87, 2006.

SIEVERDING, E. Vesicular-arbuscular mycorrhiza management in tropical agrosystems. Eschborn: Deutsche Gesellschaft für Technische Zusammenarbeit, 1991. $371 \mathrm{p}$.

SIQUEIRA, J. O. et al. Micorrizas e a degradação do solo: caracterização, efeitos e ação recuperadora. Tópicos em Ciência do Solo, Viçosa, MG, v. 5, p. 219-306, 2007.

SIQUEIRA, J. O.; COLOZZI-FILHO, A. Micorrizas vesículo-arbusculares em mudas de cafeeiro II. Efeito do fósforo no estabelecimento e funcionamento da simbiose. Revista Brasileira de Ciência do Solo, Campinas, v. 10, p. 207-211, 1986.

SMITH, S. E.; READ, D. J. Mycorrhizal Symbiosis. 3rd ed. London: Academy Press, 2008. 787 p.

SOARES, C. R. F. S.; CARNEIRO, M. A. C. Micorrizas arbusculares na recuperação de áreas degradadas. In: SIQUEIRA, J. O. et al. (Eds.). Micorrizas: 30 anos de pesquisa no Brasil. Lavras: Editora UFLA, 2010. p. 441-474.

THAPAR, H. S.; VIJYAN, A. K.; UNIYAL, K. Vesicular-arbuscular mycorrhizal associations and root colonization in some important tree species. The Indian Forester, Uttarakhand, v. 118, n. 3, p. 207-212, 1992. 
TÓTOLA, M. R.; BORGES, A. C. Growth and nutritional status of brazilian wood species Cedrella fissilis and Anadenanthera peregrina in bauxite spoil in response to arbuscular mycorrhizal inoculation and substrate amendment. Brazilian Journal of Microbiology, São Paulo, v. 31, p. 257-265, 2000. 